\title{
BANCOS DE MACRÓFITAS AQUÁTICAS COMO LOCAIS DE DESENVOLVIMENTO DAS FASES INICIAIS DE PEIXES EM VÁRZEA DO BAIXO AMAZONAS
}

\author{
Lucas Silva de Oliveira ${ }^{*}$, Ruineris Almada Cajado ${ }^{1}$, Luan Robson Bentes dos Santos ${ }^{1}$, \\ Maria Aparecida de Lima Suzukil \& Diego Maia Zacardi ${ }^{1}$
}

${ }^{1}$ Universidade Federal do Oeste do Pará, Instituto de Ciências e Tecnologia das Águas, Laboratório de Ecologia do Ictioplâncton e Pesca em Águas Interiores, Rua Vera Paz, S/N, Bloco 11, CEP 68035-110, Santarém, PA, Brasil.

E-mails: lucasmdcpa@gmail.com (*corresponding author); ruineris.cajado@gmail.com; luan.robson99@gmail.com; cidaatm@hotmail.com;dmzacardi@hotmail.com.

\begin{abstract}
Resumo: O estudo descreve a composição e estrutura da comunidade de larvas e juvenis de peixes associada a bancos de macrófitas aquáticas e avalia a importância dessas vegetações para o desenvolvimento dos estágios iniciais de vida da ictiofauna em uma ilha aluvial no Baixo Amazonas, Pará, Brasil. As amostragens foram realizadas durante os momentos de cheia/vazante de 2017 (ciclo I) e enchente de 2018 (ciclo II) em bancos de macrófitas por meio de um peneirão (malha $500 \mu \mathrm{m}$ ). Em laboratório os indivíduos foram identificados e classificados quanto ao seu estágio de desenvolvimento e verificada a abundância, a composição das espécies e similaridade entre os ciclos de amostragens. Uma análise de variância e teste t foram utilizados para constatar diferenças das densidades entre os estágios de desenvolvimento e ciclos hidrológicos, respectivamente. Foram capturados 2.193 indivíduos, dos quais $87,91 \%$ ainda em fase larval e 12,08 \% juvenil, classificados em 84 táxons e distribuídos em nove ordens, 24 famílias, 50 gêneros e 72 espécies. Durante o ciclo I, houve maior predominância de indivíduos juvenis de espécies de pequeno porte, que possuem importante função na transferência de energia no ecossistema aquático, enquanto no ciclo II os maiores valores de abundância foram registrados para as espécies de interesse econômico e de médio porte capturadas em estágios iniciais de desenvolvimento. Não foi observada diferença significativa na densidade de indivíduos entre os ciclos de amostragem, porém através da análise de similaridade foi registrada uma variação na composição de espécies entre os ciclos. A maior riqueza de espécies, dominância e densidade de indivíduos foram registradas durante a enchente. A cheia/vazante apresentou maiores valores de equitabilidade e diversidade de espécies. Assim, a comunidade de larvas e juvenis de peixes em vegetação aquática em várzea do Baixo Amazonas demonstra ser influenciada pelo ciclo hidrológico, garantindo a manutenção e renovação da ictiofauna.
\end{abstract}

Palavras-chave: Diversidade; ictiofauna; vegetação flutuante; inundação; Amazônia Oriental.

AQUATICMACROPHYTE STANDS AS EARLY STAGES OF FISH DEVELOPMENT SITES INTHE FLOODPLAIN OF THE LOWER AMAZON: The study describes the composition and structure of the larval and juvenile fishes' community associated with aquatic macrophyte stands and it evaluates the importance of this vegetation for the development of early life stages of the ichthyofauna in an alluvial island in the Lower Amazon River, state of Pará, Brazil. The sampling was conducted during the phases of full flood/receding water of 2017 (cycle I) and rising water of 2018 (cycle II) in aquatic macrophyte stands using a fishing sieve (500 $\mu \mathrm{m}$ mesh). In the laboratory, specimens were identified and classified for their development stage and verified the species composition, abundance and similarity between the sampling cycles. Variance analysis 
and t-test were used to identify possible differences in the density between the development stages and the hydrological cycles, respectively. There were captured 2,193 individuals, from which $87.91 \%$ were at the larval stage and $12.08 \%$ at the juvenile, classified in 84 taxa and identified in nine orders, 24 families, 50 genera and 72 species. During the cycle I, there was a higher predominance of juvenile individuals of small species, which have an important role in energy transferring in the aquatic ecosystem, while in the cycle II the highest values of abundance were recorded from the medium-sized species with economic importance, captured in the early development stages. No significant difference was observed in the density of individuals among the sampling cycles however, the similarity analysis registered a variation on the composition of species between the cycles. The higher species richness, dominance and density of individuals were recorded during the rising water, while the full flood/receding water presented higher values of evenness and species diversity. Thus, larval and juvenile fishes' community in aquatic vegetation in varzea in the Lower Amazon region is shown to be influenced by the hydrological cycle, ensuring the maintenance and renewal of the ichthyofauna.

Keywords: Diversity; ichthyofauna; floating vegetation; flooding; Eastern Amazon.

\section{INTRODUÇÃO}

As várzeas são ambientes altamente produtivos constituídos por áreas inundáveis periodicamente pelos rios de águas brancas. A complexidade estrutural e a elevada quantidade de nutrientes presentes (Hurd et al. 2016, Arias et al. 2018) fornecem base e sustentação para a alta produção e diversidade de peixes. Estes ambientes contribuem para o desenvolvimento de uma intensa atividade pesqueira (comercial e de subsistência) na região amazônica (Batista et al. 1998, Saint-Paul et al. 2000, Goulding et al. 2018), que possui um dos maiores consumos per capita de pescado (169 kg/ ano) do mundo em comunidades ribeirinhas (Isaac et al. 2015).

Um dos componentes fundamentais para a manutenção desses ambientes é a presença de macrófitas aquáticas, as quais desempenham um importante papel nos sistemas rios-planícies de inundação. São responsáveis pela formação de micro-habitats, pela complexidade biológica, pelas relações intra e interespecíficas e de conservação de grande parte da diversidade de peixes presente nas áreas marginais de canais e lagos da várzea (Zacardi 2014, Pinheiro et al. 2016). Com relação a este último aspecto, Agostinho et al. (2002) relatam maiores valores do índice de Shannon e de riqueza de espécies de peixes na interface entre as macrófitas e as regiões pelágicas de ambientes lênticos, com predomínio de organismos de menor porte nas bordas dos bancos de vegetação.

As partes submersas dessas vegetações aquáticas são utilizadas pela ictiofauna, em suas diferentes fases do ciclo de vida, como importantes locais para o desenvolvimento, manutenção e renovação das espécies de peixes (Sánchez-Botero \& Araújo-Lima 2001, Soares et al. 2014, Pinheiro et al. 2016). Amostragens realizadas em bancos de macrófitas são pouco exploradas nos levantamentos ictiofaunísticos, uma que vez requerem a utilização de diferentes apetrechos e metodologias de coletas a fim de melhor descrever a composição das espécies (Gomes et al. 2012, Affonso et al. 2015).

Embora alguns estudos de composição, abundância e distribuição espaço-temporal da ictiofauna associada a bancos de macrófitas aquáticas na região amazônica tenham sido realizados a fim de verificar a relação entre a ecologia de peixes e os bancos de macrófitas (Soares et al. 1986, Henderson \& Hamilton 1995, Sanchez-Botero \& Araújo-Lima 2001, SanchezBotero et al. 2003, Schiezari et al. 2003, Petry et al. 2003, Sanchez-Botero et al. 2008, Prado et al. 2009, Prado et al. 2010, Soares et al. 2014, Magalhães et al. 2015, Pinheiro et al. 2016), a importância desse habitat para a biodiversidade de peixes, principalmente nos primeiros estágios de vida, ainda é um aspecto pouco explorado na região de várzea tropical do Baixo Amazonas.

Dessa forma, o trabalho descreve a composição e estrutura dos estágios iniciais da ictiofauna associada a bancos de vegetações aquáticas na ilha das Marrecas, no trecho inferior do rio Amazonas. Esse conhecimento é uma ferramenta útil para inferir os locais de desenvolvimento e crescimento de larvas e juvenis de peixes, aspecto 
de grande importância para a legislação pesqueira e conservação dos recursos naturais na Amazônia.

\section{MATERIAL E MÉTODOS}

\section{Área de estudo}

A ilha das Marrecas está situada no canal principal do trecho inferior do rio Amazonas e permanece conectada ao longo de todo o ano com o sistema fluvial. Está localizada entre os quadrantes dispostos nas latitudes $2^{\circ} 12^{\prime} 41,86^{\prime \prime} \mathrm{S}$ e $2^{\circ} 18^{\prime} 52,90^{\prime \prime}$ S e longitudes $54^{\circ} 45^{\prime} 42,49^{\prime \prime}$ W e $54^{\circ} 43^{\prime} 11,89^{\prime \prime} \mathrm{W}$, próximo a cidade de Santarém, na região do Baixo Amazonas, Pará (Figura 1). A região é composta por uma extensa área de várzea, com predominância de bancos de Paspalum repens PJ Bergius (Poaceae), e outras espécies de macrófitas flutuantes em baixas abundâncias como Eichhornia spp. (Pontederiaceae), Pistia stratiotes L. (Araceae) e Salvinia spp. (Salviniaceae) com ocorrência registrada apenas nas fases de inundação, pois durante o período de seca hidrológica grande parte dessas plantas aquáticas entram em senescência.

\section{Coleta do material biológico}

As coletas foram realizadas em bancos de macrófitas aquáticas que crescem nas margens de lagos e canais de inundação da ilha, durante dois ciclos de amostragens representando momentos distintos de inundação do período hidrológico de 2017 e 2018, mediante autorização de coleta de número 72.330 emitida pelo SISBIO/ICMBio/MMA. O ciclo I ocorreu durante os meses de maio, junho e julho de 2017 representando o pico da cheia e início da vazante deste ano e o ciclo II em janeiro, fevereiro e março de 2018, momento de enchente do rio Amazonas na região.

As amostragens foram realizadas mensalmente durante o período diurno e distribuídas em seis locais de coleta, com distâncias de aproximadamente 40 metros entre si. Para a obtenção do material biológico utilizou-se técnica de captura ativa por meio de um apetrecho de pesca denominado peneirão, descrito por Nakatani et al. (2001) e utilizada por Pinheiro et al. (2016). $O$ peneirão é uma rede presa a um aro retangular por intermédio de uma lona (malha de $500 \mu \mathrm{m}$ ),

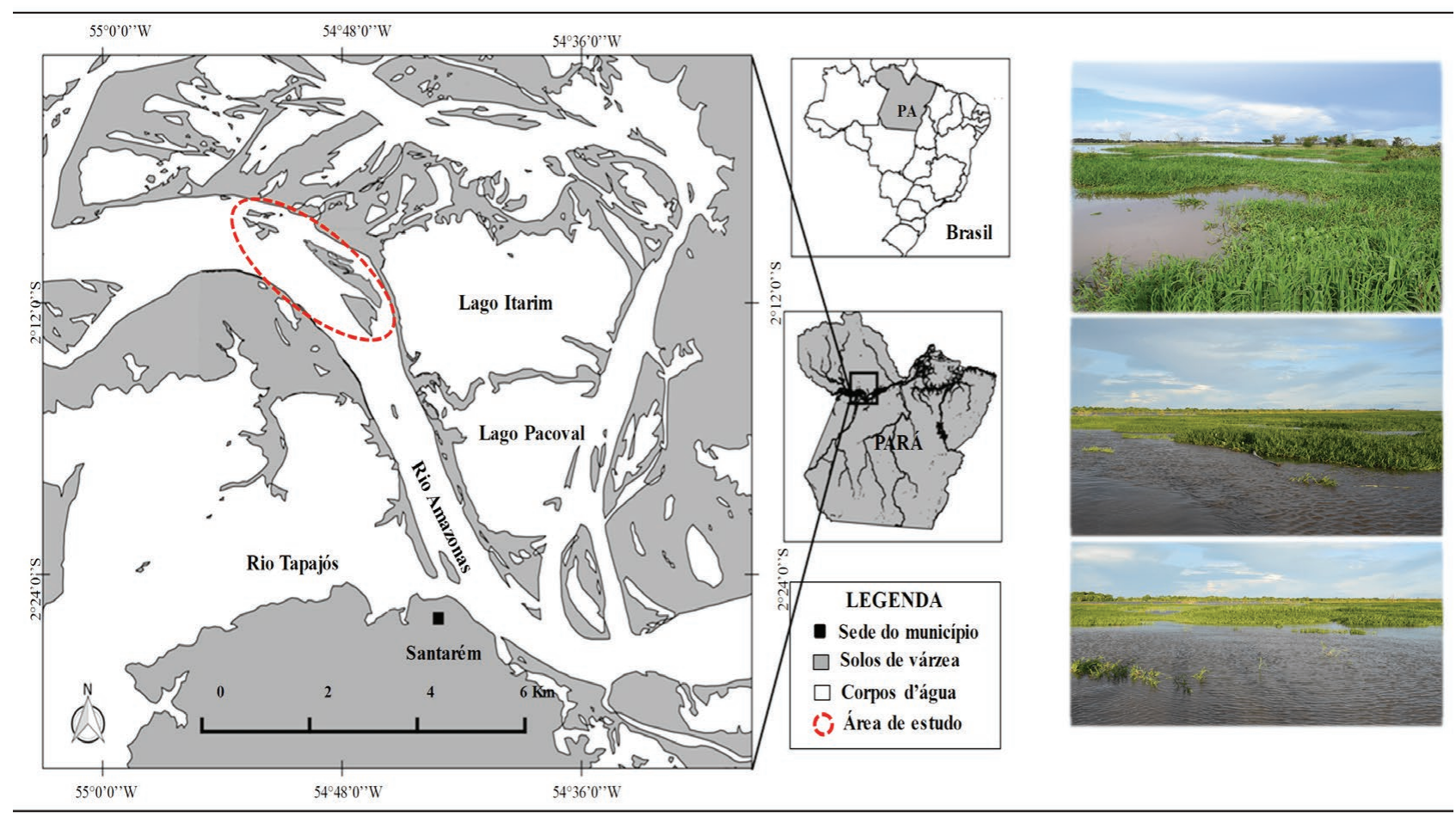

Figura 1: Localização da área de estudo, ilha das Marrecas, situada no trecho baixo do rio Amazonas, próximo à Santarém (Pará-Brasil), região de várzea dominada por diversos estandes de macrófitas aquáticas durante os momentos de inundação.

Figure 1: Study area, Ilha das Marrecas, in the Lower Amazon River, close to Santarém (state of Pará-Brazil), floodplain region dominated by many aquatic macrophyte stands during the flood. 
com 1,0 metro de comprimento, por 1,5 metros de largura e 1,0 metro de altura. Esse apetrecho foi introduzido embaixo da vegetação aquática (50 $\mathrm{cm}$ de profundidade) e levantado rapidamente, procedendo-se em seguida à retirada e separação cuidadosa das larvas e juvenis de peixes do material vegetal e detritos.

Em cada local amostrado foram efetuados cinco lances consecutivos do peneirão em bancos mistos, de tamanhos e formatos aleatórios. Este procedimento teve o intuito de obter a melhor representatividade possível da comunidade de larvas e juvenis de peixes, sendo mantido o mesmo esforço e número de repetições ao longo dos meses, totalizando 18 amostras por ciclo e 36 amostras ao final do estudo.

Após serem capturados, as larvas e os juvenis foram submetidos imediatamente à solução de eugenol $(50 \mathrm{mg} / \mathrm{L})$. Em seguida, acondicionados em frascos de polietileno com capacidade de 500 $\mathrm{mL}$, devidamente etiquetados, contendo formalina a $10 \%$ como fixador e transportados para posterior análise em laboratório.

\section{Procedimentos em laboratório}

As larvas foram classificadas quanto aos estágios de desenvolvimento em pré-flexão, flexão e pósflexão, utilizando-se como critério principal o nível de flexão da estrutura final da notocorda, formação da nadadeira caudal e seus elementos de suporte, conforme proposto por Nakatani et al. (2001) e Orsi et al. (2016). Posteriormente foram quantificadas e identificadas até o menor nível taxonômico possível de acordo com Araújo-Lima \& Donald (1988), Araújo-Lima et al. (1993) e para grandes grupos, como ordens e famílias utilizou-se Nakatani et al. (2001) e Orsi et al. (2016).

Os indivíduos considerados juvenis foram classificados por apresentarem completa formação e segmentação dos raios das nadadeiras e surgimento das escamas (Nakatani et al. 2001), entretanto com gônadas imaturas. Estes indivíduos foram quantificados, identificados e transferidos para potes contendo álcool a $70 \%$ para conservação. A identificação dos espécimes neste estágio foi realizada com o auxílio de chaves taxonômicas contidas em Géry (1977), Ferreira et al. (1998), Santos et al. (2004), Santos et al. (2006), Britski et al. (2007) e Soares et al. (2011), e algumas espécies confirmadas por especialistas. O enquadramento taxonômico foi baseado em Betancur-R et al. (2017). A estratégia reprodutiva (migrador e não migrador) das espécies foi baseada de acordo com as informações dos indivíduos adultos (Goulding 1980, Barthem \& Fabré 2004, Granado-Lourencio et al.2005, Soares et al. 2008). Espécimes-testemunho foram depositados no Laboratório de Ecologia do Ictioplâncton e Pesca em Águas Interiores da Universidade Federal do Oeste do Pará para constituir uma coleção de referência.

\section{Análises de dados}

Foi calculada a abundância relativa das larvas e juvenis de peixes através da fórmula 1 , em que: $\mathrm{N}$ = número de indivíduos do grupo taxonômico e $\mathrm{T}=$ número total de indivíduos capturados.

$$
\operatorname{Ar}=\left(N^{*} 100\right) / \mathrm{T}
$$

Enquanto que a padronização da densidade de indivíduos por $\mathrm{m}^{3}$ foi realizada por meio da fórmula 2 modificado de Nakatani et al. (2001), em que Z é o número de indivíduos por $\mathrm{m}^{3}$, $\mathrm{X}$ é o número de indivíduos coletados, $\mathrm{A}=$ área do peneirão $\left(\mathrm{m}^{2}\right)$ e P a profundidade $(\mathrm{m})$ em que os lances foram realizados.

\section{$\mathrm{Z}=\mathrm{X} /\left(\mathrm{A}^{*} \mathrm{~L}^{*} \mathrm{P}\right)$}

A análise de similaridade foi realizada com os dados de abundância total das espécies ao longo dos meses amostrais utilizando-se o coeficiente de Bray-Curtis e o método de ligação UPGMA, com o objetivo de evidenciar padrões de similaridade na composição de espécies ao longo dos meses de coletas. A riqueza de espécies foi considerada como o número de espécies encontradas em cada ciclo de amostragem. Para determinar a diversidade, utilizou-se o índice de Shannon-Winner (H') e, com bases nas abundâncias totais, foram estimadas a equitabilidade de Pielou (J') e a dominância de espécies (D) (Magurran 2003), utilizando-se apenas os indivíduos identificados em nível de gênero e espécie.

Os pressupostos de normalidade e homocedasticidade foram avaliados usando o teste de Shapiro-Wilk e Levene, respectivamente. Para verificar possíveis diferenças dos índices ecológicos (diversidade, dominância, equitabilidade e riqueza de espécies) entre os 
ciclos de amostragem, foi realizado um teste $t$ de student (Zar 1999). A análise de variância (ANOVA One Way) foi aplicada para investigar possíveis diferenças entre a densidade dos estágios de desenvolvimento ao longo dos meses de amostragens e, sempre que observada diferenças significativas $(p<0,05)$, foi aplicado teste de Tukey (Winer et al. 1991). As análises foram realizadas no ambiente R 3.5.2 (R Core Team, 2018) utilizando o pacote Vegan (Oksanen et al. 2018).

\section{RESULTADOS}

Foram capturados 2.193 indivíduos ao longo dos dois ciclos de amostragens, desse total 1.928 encontravam-se em estágios larvais (pré-flexão: 63,74 \%, flexão: 24,33 \% e pós-flexão: 11,93 \%) e 265 foram classificados como juvenis. No primeiro ciclo, foram registrados 82 larvas e 133 juvenis, enquanto no segundo ciclo, foram registrados 1.846 larvas e 132 juvenis. Os espécimes foram classificados em 84 táxons, distribuídos em nove ordens, 24 famílias, 50 gêneros e 72 espécies (Tabela 1). Deste total, a ordem Characiformes predominou com maior número de indivíduos nos dois períodos $(69,76 \%$ no ciclo I e $94,56 \%$ no ciclo II), perfazendo $92,11 \%$ dos organismos capturados durante o estudo, seguida por Clupeiformes e Siluriformes.

As famílias mais representativas em número de indivíduos, em ordem decrescente de abundância, foram Serrasalmidae, Triportheidae, Hemiodontidae, Anostomidae e Characidae, totalizando juntas mais de $90 \%$ dos indivíduos ao longo dos ciclos analisados (Figura 2). Enquanto em relação a riqueza de espécies foram

Tabela 1: Composição taxonômica classificada em ordem, família e espécie. Número de indivíduos (N), abundância relativa (AR) e importância econômica (IE) na região (pesca comercial e de subsistência - † e ornamental - $\diamond$ ). Estratégia reprodutiva da espécie (ER): M (migrador) e NM (não migrador).

Table 1: Taxonomic composition classified in order, family and species. Number of individuals (N), relative abundance (AR) and economic importance (IE) in the region (commercial and subsistence fishing - + and ornamental fishing - $\diamond$ ). Reproductive strategy of the species (ER): M (migrant) e NM (non-migrant).

\begin{tabular}{|c|c|c|c|c|c|c|c|c|}
\hline \multirow{2}{*}{$\begin{array}{l}\text { Ordem } \\
\text { Família } \\
\text { Espécie }\end{array}$} & \multicolumn{2}{|c|}{ Ciclo I } & \multicolumn{2}{|c|}{ Ciclo II } & \multirow[t]{2}{*}{ IE } & \multirow[t]{2}{*}{ ER } & \multicolumn{2}{|c|}{$\underset{(\mathbf{m m})}{\operatorname{Tamanho}}$} \\
\hline & $\mathbf{N}$ & AR (\%) & $\mathbf{N}$ & AR (\%) & & & Mín. & Máx. \\
\hline Characiformes & 1 & 0,47 & 29 & 1,47 & & & 4,5 & 8 \\
\hline \multicolumn{9}{|l|}{ Anostomidae } \\
\hline $\begin{array}{l}\text { Anostomoides laticeps (Eigenmann, } \\
\text { 1912) }\end{array}$ & 0 & 0 & 5 & 0,25 & $\dagger$ & M & 19 & 42 \\
\hline Leporinus fasciatus (Bloch, 1794) & 0 & 0 & 29 & 1,47 & $\diamond$ & M & 13 & 38 \\
\hline Leporinus sp. & 0 & 0 & 3 & 0,15 & $\dagger$ & & 11 & 13 \\
\hline Rhytiodus microlepis Kner, 1858 & 14 & 6,51 & 13 & 0,66 & $\dagger$ & M & 11 & 78 \\
\hline $\begin{array}{l}\text { Schizodon fasciatus Spix \& Agassiz, } \\
1829\end{array}$ & 0 & 0 & 23 & 1,16 & $\dagger$ & M & 5 & 17 \\
\hline Schizodon sp. & 0 & 0 & 13 & 0,66 & $\dagger$ & & 5,2 & 10 \\
\hline Characidae & 5 & 2,33 & 4 & 0,2 & & & 5 & 11 \\
\hline Aphyocharax alburnos (Günther, 1869) & 23 & 10,7 & 4 & 0,2 & $\diamond$ & NM & 11 & 35 \\
\hline $\begin{array}{l}\text { Aphyocharax nattereri (Steindachner, } \\
\text { 1882) }\end{array}$ & 3 & 1,4 & 0 & 0 & $\diamond$ & NM & 12 & 20 \\
\hline Hemigrammus levis Durbin, 1908 & 0 & 0 & 1 & 0,05 & $\diamond$ & NM & - & 12 \\
\hline $\begin{array}{l}\text { Hemigrammus ocellifer (Steindachner, } \\
\text { 1882) }\end{array}$ & 1 & 0,47 & 0 & 0 & $\diamond$ & NM & - & 16 \\
\hline Hemigrammus sp. & 4 & 1,86 & 0 & 0 & & NM & 12 & 17 \\
\hline Heterocharax sp. & 0 & 0 & 2 & 0,1 & & NM & 25 & 24 \\
\hline
\end{tabular}


Tabela 1: ...Continuação

Table 1:...Continued

\begin{tabular}{|c|c|c|c|c|c|c|c|c|}
\hline \multirow{2}{*}{$\begin{array}{l}\text { Ordem } \\
\text { Família } \\
\text { Espécie }\end{array}$} & \multicolumn{2}{|c|}{ Ciclo I } & \multicolumn{2}{|c|}{ Ciclo II } & \multirow[t]{2}{*}{ IE } & \multirow[t]{2}{*}{ ER } & \multicolumn{2}{|c|}{$\begin{array}{c}\text { Tamanho } \\
(\mathbf{m m})\end{array}$} \\
\hline & $\mathbf{N}$ & AR (\%) & $\mathbf{N}$ & AR (\%) & & & Mín. & Máx. \\
\hline $\begin{array}{l}\text { Hyphessobrycon cf. diancistrus } \\
\text { Weitzman, } 1977\end{array}$ & 1 & 0,47 & 1 & 0,05 & $\diamond$ & NM & 18 & 23 \\
\hline $\begin{array}{l}\text { Moenkhausia celibela Marinho \& } \\
\text { Langeani, } 2010\end{array}$ & 2 & 0,93 & 0 & 0 & $\diamond$ & NM & 14,5 & 15 \\
\hline Moenkhausia lepidura (Kner, 1858) & 6 & 2,79 & 0 & 0 & $\diamond$ & NM & 15 & 20 \\
\hline Monkhausia dicroura (Kner, 1858) & 2 & 0,93 & 1 & 0,05 & $\diamond$ & NM & 23 & 34 \\
\hline Odontostilbe cf. fugitiva Cope, 1870 & 1 & 0,47 & 0 & 0 & & NM & - & 11 \\
\hline Prionobrama filigera (Cope, 1870) & 29 & 13,49 & 1 & 0,05 & $\diamond$ & NM & 10 & 32 \\
\hline $\begin{array}{l}\text { Serrapinnus cf microdon (Eigenmann, } \\
\text { 1915) }\end{array}$ & 0 & 0 & 1 & 0,05 & & NM & - & 27 \\
\hline \multicolumn{9}{|l|}{ Cynodontidae } \\
\hline $\begin{array}{l}\text { Rhapiodon vulpinis Spix \& Agassiz, } \\
1829\end{array}$ & 0 & 0 & 1 & 0,05 & $\dagger$ & M & - & 36 \\
\hline \multicolumn{9}{|l|}{ Erythrinidae } \\
\hline Hoplias malabaricus (Bloch, 1794) & 2 & 0,93 & 7 & 0,35 & $\diamond \dagger$ & NM & 8,5 & 27 \\
\hline \multicolumn{9}{|l|}{ Gasteropelecidae } \\
\hline $\begin{array}{l}\text { Thoracocharax securis (De Filippi, } \\
\text { 1853) }\end{array}$ & 0 & 0 & 2 & 0,1 & $\diamond$ & NM & - & 10 \\
\hline Thoracocharax sp. & 1 & 0,47 & 0 & 0 & & & - & 19 \\
\hline Hemiodontidae & 0 & 0 & 21 & 1,06 & & & 3 & 8 \\
\hline Anodus elongatus Agassiz, 1829 & 0 & 0 & 23 & 1,16 & $\diamond \dagger$ & M & 5 & 13,5 \\
\hline Hemiodus immaculatus Kner, 1858 & 0 & 0 & 5 & 0,25 & $\dagger$ & M & 5 & 9 \\
\hline Hemiodus microlepis Kner, 1858 & 0 & 0 & 157 & 7,94 & $\dagger$ & M & 4 & 15 \\
\hline Hemiodus sp. & 0 & 0 & 1 & 0,05 & $\dagger$ & & 3,5 & 9 \\
\hline Serrasalmidae & 0 & 0 & 2 & 0,1 & & & 5,5 & 6 \\
\hline $\begin{array}{l}\text { Colossoma macropomum (Cuvier, } \\
\text { 1816) }\end{array}$ & 2 & 0,93 & 0 & 0 & $\dagger$ & M & - & 9 \\
\hline Metynnis lippincottianus (Cope, 1870) & 2 & 0,93 & 0 & 0 & $\diamond \dagger$ & M & 12 & 29 \\
\hline $\begin{array}{l}\text { Myloplus cf. rubripinnis (Müller \& } \\
\text { Troschel, 1844) }\end{array}$ & 8 & 3,72 & 2 & 0,1 & $\diamond$ & NM & 13 & 20 \\
\hline $\begin{array}{l}\text { Mylossoma aureum (Spix \& Agassiz, } \\
\text { 1829) }\end{array}$ & 8 & 3,72 & 676 & 34,18 & $\dagger$ & M & 5 & 47 \\
\hline Mylossoma albiscopum (Cope, 1872) & 18 & 8,37 & 276 & 13,95 & $\dagger$ & M & 5 & 41 \\
\hline Serrasalmus maculatus Kner, 1858 & 0 & 0 & 11 & 0,56 & $\diamond \dagger$ & NM & 13 & 26 \\
\hline $\begin{array}{l}\text { Serrasalmus rhombeus (Linnaeus, } \\
\text { 1766) }\end{array}$ & 9 & 4,19 & 2 & 0,1 & $\diamond \dagger$ & NM & 8 & 25 \\
\hline Serrasalmus sp. 1 & 0 & 0 & 8 & 0,4 & $\dagger$ & & 8 & 14 \\
\hline Serrasalmus spilopleura Kner, 1858 & 7 & 3,26 & 0 & 0 & $\diamond \dagger$ & NM & 16 & 26 \\
\hline
\end{tabular}

Tabela 1: Continua na próxima página... Table 1: Continued on next page... 
Tabela 1: ...continuação

Table 1:...Continued

\begin{tabular}{|c|c|c|c|c|c|c|c|c|}
\hline \multirow{2}{*}{$\begin{array}{l}\text { Ordem } \\
\text { Família } \\
\text { Espécie }\end{array}$} & \multicolumn{2}{|c|}{ Ciclo I } & \multicolumn{2}{|c|}{ Ciclo II } & \multirow[t]{2}{*}{ IE } & \multirow[t]{2}{*}{$\mathbf{E R}$} & \multicolumn{2}{|c|}{$\begin{array}{c}\text { Tamanho } \\
(\mathbf{m m})\end{array}$} \\
\hline & $\mathbf{N}$ & AR (\%) & $\mathbf{N}$ & AR (\%) & & & Mín. & Máx. \\
\hline \multicolumn{9}{|l|}{ Triportheidae } \\
\hline Triportheus albus Cope, 1872 & 1 & 0,47 & 0 & 0 & $\diamond \dagger$ & $\mathrm{M}$ & - & 18 \\
\hline $\begin{array}{l}\text { Triportheus angulatus (Spix \& Agassiz, } \\
\text { 1829) }\end{array}$ & 0 & 0 & 197 & 9,96 & $\diamond \dagger$ & $\mathrm{M}$ & 4,5 & 40 \\
\hline $\begin{array}{l}\text { Triportheus auritus (Valenciennes, } \\
\text { 1850) }\end{array}$ & 0 & 0 & 180 & 9,1 & $\dagger$ & $\mathrm{M}$ & 7 & 31 \\
\hline Triportheus sp.1 & 0 & 0 & 169 & 8,54 & & & 4 & 9 \\
\hline \multicolumn{9}{|l|}{ Beloniformes } \\
\hline \multicolumn{9}{|l|}{ Belonidae } \\
\hline Strongylura timucu (Walbaum, 1792) & 0 & 0 & 2 & 0,1 & & NM & 30 & 56 \\
\hline \multicolumn{9}{|l|}{ Cichliformes } \\
\hline \multicolumn{9}{|l|}{ Cichlidae } \\
\hline $\begin{array}{l}\text { Aequidens cf. plagiozonatus Kullander, } \\
1984\end{array}$ & 2 & 0,93 & 0 & 0 & $\diamond$ & NM & 20 & 35 \\
\hline Apistogramma sp. & 1 & 0,47 & 0 & 0 & & & - & 25 \\
\hline Crenicichla saxatilis (Linnaeus, 1758) & 1 & 0,47 & 0 & 0 & $\dagger$ & NM & - & 48 \\
\hline $\begin{array}{l}\text { Crenicichla semicincta Steindachner, } \\
1892\end{array}$ & 0 & 0 & 1 & 0,05 & $\dagger$ & NM & - & 76 \\
\hline Mesonauta insignis (Heckel, 1840) & 9 & 4,19 & 0 & 0 & $\diamond$ & NM & 13 & 39 \\
\hline Satanoperca sp. & 1 & 0,47 & 0 & 0 & $\dagger$ & & - & 21 \\
\hline Clupeiformes & 0 & 0 & 1 & 0,05 & & & - & 3,5 \\
\hline \multicolumn{9}{|l|}{ Clupeidae } \\
\hline $\begin{array}{l}\text { Rhinosardinia amazonica } \\
\text { (Steindachner, 1879) }\end{array}$ & 1 & 0,47 & 7 & 0,35 & & $\mathrm{M}$ & 15 & 20 \\
\hline Engraulidae & 1 & 0,47 & 31 & 1,57 & & & 5 & 20 \\
\hline $\begin{array}{l}\text { Anchoviella guianensis (Eigenmann, } \\
\text { 1912) }\end{array}$ & 0 & 0 & 2 & 0,1 & & $\mathrm{M}$ & 13 & 23 \\
\hline Anchoviella sp. & 0 & 0 & 10 & 0,51 & & $\mathrm{M}$ & 13 & 26 \\
\hline Lycengraulis batesii (Günther, 1868) & 0 & 0 & 2 & 0,1 & & $\mathrm{M}$ & 28 & 47 \\
\hline \multicolumn{9}{|l|}{ Pristigasteridae } \\
\hline $\begin{array}{l}\text { Pellona castelnaeana Valenciennes, } \\
1847\end{array}$ & 0 & 0 & 4 & 0,2 & $\dagger$ & $\mathrm{M}$ & 7 & 28 \\
\hline $\begin{array}{l}\text { Pellona flavipinnis (Valenciennes, } \\
\text { 1837) }\end{array}$ & 1 & 0,47 & 4 & 0,2 & $\dagger$ & $\mathrm{M}$ & 8 & 23 \\
\hline \multicolumn{9}{|l|}{ Cyprinodontiformes } \\
\hline \multicolumn{9}{|l|}{ Poeciliidae } \\
\hline $\begin{array}{l}\text { Pamphorichthys scalpridens (Garman, } \\
\text { 1895) }\end{array}$ & 22 & 10,23 & 4 & 0,2 & $\diamond$ & NM & 7 & 22 \\
\hline Rivulidae & 0 & 0 & 1 & 0,05 & & & & \\
\hline Rivulus sp. & 5 & 2,33 & 0 & 0 & & NM & 21 & 39 \\
\hline
\end{tabular}


Tabela 1: ...Continuação

Table 1:...Continued

\begin{tabular}{|c|c|c|c|c|c|c|c|c|}
\hline \multirow{2}{*}{$\begin{array}{l}\text { Ordem } \\
\text { Família } \\
\text { Espécie }\end{array}$} & \multicolumn{2}{|c|}{ Ciclo I } & \multicolumn{2}{|c|}{ Ciclo II } & \multirow[t]{2}{*}{ IE } & \multirow{2}{*}{ ER } & \multicolumn{2}{|c|}{$\begin{array}{l}\text { Tamanho } \\
(\mathbf{m m})\end{array}$} \\
\hline & $\mathbf{N}$ & AR (\%) & $\mathbf{N}$ & AR (\%) & & & Mín. & Máx. \\
\hline \multicolumn{9}{|l|}{ Gymnotiformes } \\
\hline \multicolumn{9}{|l|}{ Hypopomidae } \\
\hline $\begin{array}{l}\text { Brachyhypopomus regani (Garman, } \\
\text { 1895) }\end{array}$ & 1 & 0,47 & 0 & 0 & $\diamond$ & NM & - & 35 \\
\hline \multicolumn{9}{|l|}{ Sternopygidae } \\
\hline $\begin{array}{l}\text { Eigenmannia aff. virescens } \\
\text { (Valenciennes, 1836) }\end{array}$ & 1 & 0,47 & 0 & 0 & $\diamond$ & NM & 42 & 47 \\
\hline Siluriformes & 0 & 0 & 2 & 0,1 & & & 4 & 5 \\
\hline Auchinipteridae & 0 & 0 & 1 & 0,05 & & & - & 7 \\
\hline Auchenipterus sp. & 1 & 0,47 & 0 & 0 & & & - & 15 \\
\hline Cetopsidae & 0 & 0 & 1 & 0,05 & & & - & 17 \\
\hline $\begin{array}{l}\text { Cetopsis coecutiens (Lichtenstein, } \\
\text { 1819) }\end{array}$ & 0 & 0 & 1 & 0,05 & $\diamond$ & NM & - & 7 \\
\hline Tatia sp. & 0 & 0 & 1 & 0,05 & & & - & 15 \\
\hline $\begin{array}{l}\text { Trachyleopterus galeatus (Linnaeus, } \\
\text { 1766) }\end{array}$ & 0 & 0 & 1 & 0,05 & $\diamond$ & NM & - & 11 \\
\hline Doradidae & 0 & 0 & 1 & 0,05 & & & - & 8 \\
\hline Nemadoras humeralis (Kner, 1855) & 2 & 0,93 & 0 & 0 & $\diamond$ & NM & 9 & 31 \\
\hline Loricariidae & 0 & 0 & 1 & 0,05 & & & - & 13 \\
\hline Ancistrus sp. & 0 & 0 & 1 & 0,05 & & & - & 16 \\
\hline $\begin{array}{l}\text { Hypoptopoma elongatum Aquino \& } \\
\text { Schaefer, } 2010\end{array}$ & 0 & 0 & 13 & 0,66 & $\diamond$ & NM & 15 & 34 \\
\hline Hypoptopoma sp.1 & 1 & 0,47 & 3 & 0,15 & & & 13 & 23 \\
\hline Hypostomus sp. & 1 & 0,47 & 0 & 0 & & & - & 16 \\
\hline $\begin{array}{l}\text { Pterygoplichthys pardalis (Castelnau, } \\
\text { 1855) }\end{array}$ & 1 & 0,47 & 1 & 0,05 & $\diamond \dagger$ & NM & 31 & 91 \\
\hline \multicolumn{9}{|l|}{ Pimelodidae } \\
\hline Pimelodus blochii Valenciennes, 1840 & 0 & 0 & 1 & 0,05 & $\diamond \dagger$ & M & - & 9,1 \\
\hline Pimelodus sp.1 & 0 & 0 & 5 & 0,25 & & & - & 13 \\
\hline $\begin{array}{l}\text { Sorubim lima (Bloch \& Schneider, } \\
\text { 1801) }\end{array}$ & 0 & 0 & 1 & 0,05 & $\dagger$ & M & - & 9 \\
\hline \multicolumn{9}{|l|}{ Synbranchiformes } \\
\hline \multicolumn{9}{|l|}{ Synbranchidae } \\
\hline Synbranchus marmoratus Bloch, 1795 & 12 & 5,58 & 1 & 0,05 & & NM & 30 & 81 \\
\hline \multicolumn{9}{|l|}{ Tetraodontiformes } \\
\hline \multicolumn{9}{|l|}{ Tetraodontidae } \\
\hline $\begin{array}{l}\text { Colomesus asellus (Müller \& Troschel, } \\
\text { 1849) }\end{array}$ & 1 & 0,47 & 4 & 0,2 & $\diamond$ & NM & 8,5 & 22 \\
\hline Total & 215 & 100 & 1978 & 100 & & & & \\
\hline
\end{tabular}




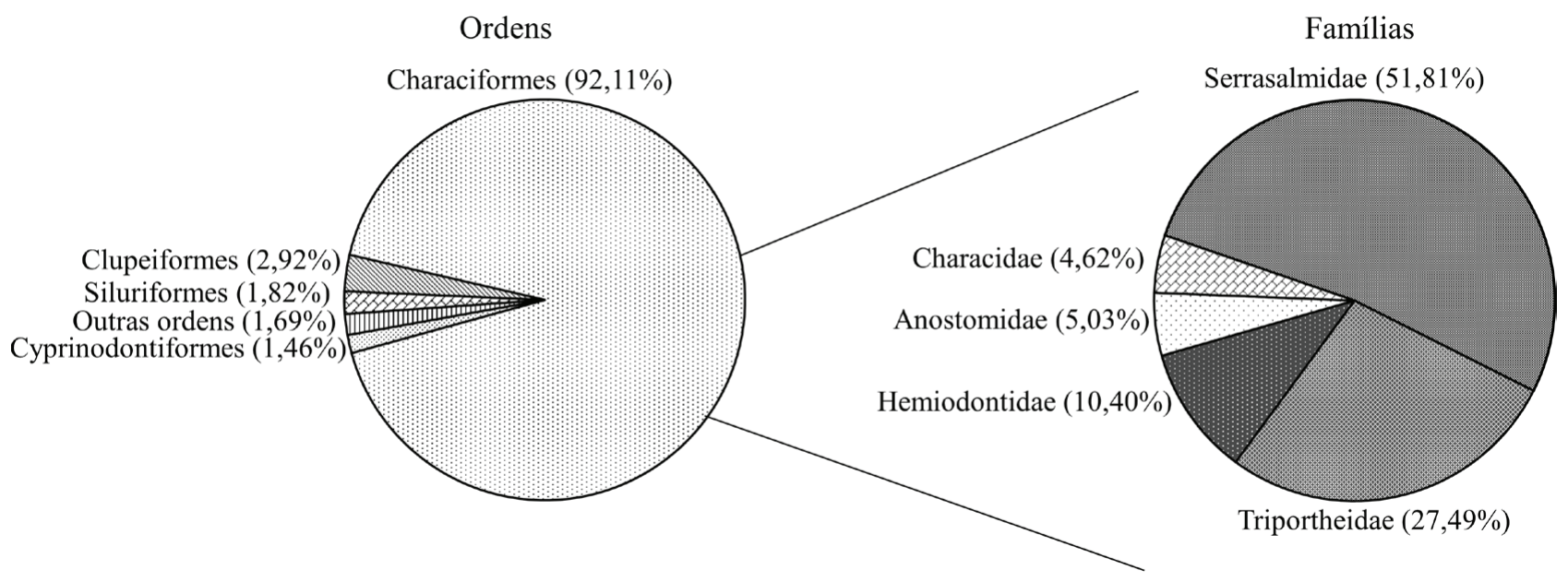

Figura 2: Abundância relativa das ordens e famílias de Characiformes (mais abundantes) associadas a vegetação aquática durante o período de estudo nas áreas alagadas da ilhas Marrecas, Baixo Amazonas, Pará.

Figure 2: Relative abundance of orders and families of Characiformes (most abundant group) associated with the aquatic vegetation during the study period in the flooded areas of Ilhas Marrecas, Lower Amazon River, state of Pará

Characidae (13), Serrasalmidae (9), Anostomidae (6) e Cichlidae (6).

Vinte e duas espécies foram exclusivas no ciclo I (pico da cheia), quarenta e dois táxons (33 espécies) ocorreram apenas no ciclo II (enchente) e dezoito espécies foram registradas em ambos os ciclos de amostragem. Characiformes (oito famílias, 23 gêneros e 40 espécies) foi a ordem mais diversa seguida por Siluriformes (cinco famílias, 11 gêneros e 13 espécies). As demais ordens, Clupeiformes e Cichliformes (seis espécies, cada), Cyprinodontiformes e Gymnotiformes (duas espécies, cada), e Synbranchiformes e Tetraodontiformes (uma espécie, cada), apresentaram pouca riqueza.

Durante o primeiro ciclo de amostragem, as maiores densidades foram observadas para as espécies de pequeno porte como Prionobrama filigera (Cope, 1870), Aphyocharax alburnos (Günther, 1869) e Pamphorichthys scalpridens (Garman, 1895) representando juntas 34,42 \% das espécies capturadas. Já no Ciclo II, os maiores valores foram registrados para as espécies de médio porte e de interesse econômico como Mylossoma aureum (Spix \& Agassiz, 1829), M. albiscopum (Cope, 1829) e Triportheus angulatus (Spix \& Agassiz, 1829), totalizando 58,09 \% dos indivíduos capturados, não sendo constatada diferença significativa da densidade de indivíduos entre os ciclos de amostragens (Teste t; $p=0,066$ ).

Por meio da análise de similaridade foi observado a formação de dois grandes grupos representados pelos ciclos de amostragens. Janeiro e fevereiro foram mais similares no segundo ciclo de coleta, enquanto junho e julho apresentaram maior similaridade no ciclo I (Figura 3).

Durante o ciclo II foi constatado maior abundância de indivíduos (Figura 4), de dominância e de riqueza de espécies em relação ao ciclo I, que por sua vez, apresentou maiores valores de equitabilidade e diversidade de espécies, ambas registradas nos meses de julho e maio, respectivamente (Tabela 2). As espécies M. aureum e T. angulatus foram responsáveis por esses altos valores, com diferenças significativas entre os índices ecológicos, com exceção do número de espécies (Tabela 2).

Com relação aos estágios de desenvolvimento, foram observadas diferenças significativas no ciclo I (ANOVA; $\mathrm{F}=15,571 ; p=0,001$ ) e para o ciclo II (ANOVA; $\mathrm{F}=5,851 ; p=0,020$ ). O estágio juvenil foi responsável por essa diferença no ciclo I, sendo significativamente distinto dos estágios de préflexão (Tukey; $p=0,001$ ) e flexão (Tukey; $p=0,002$ ). Já no ciclo II, a fase de pré-flexão foi dominante e apresentou diferença significativa entre os 


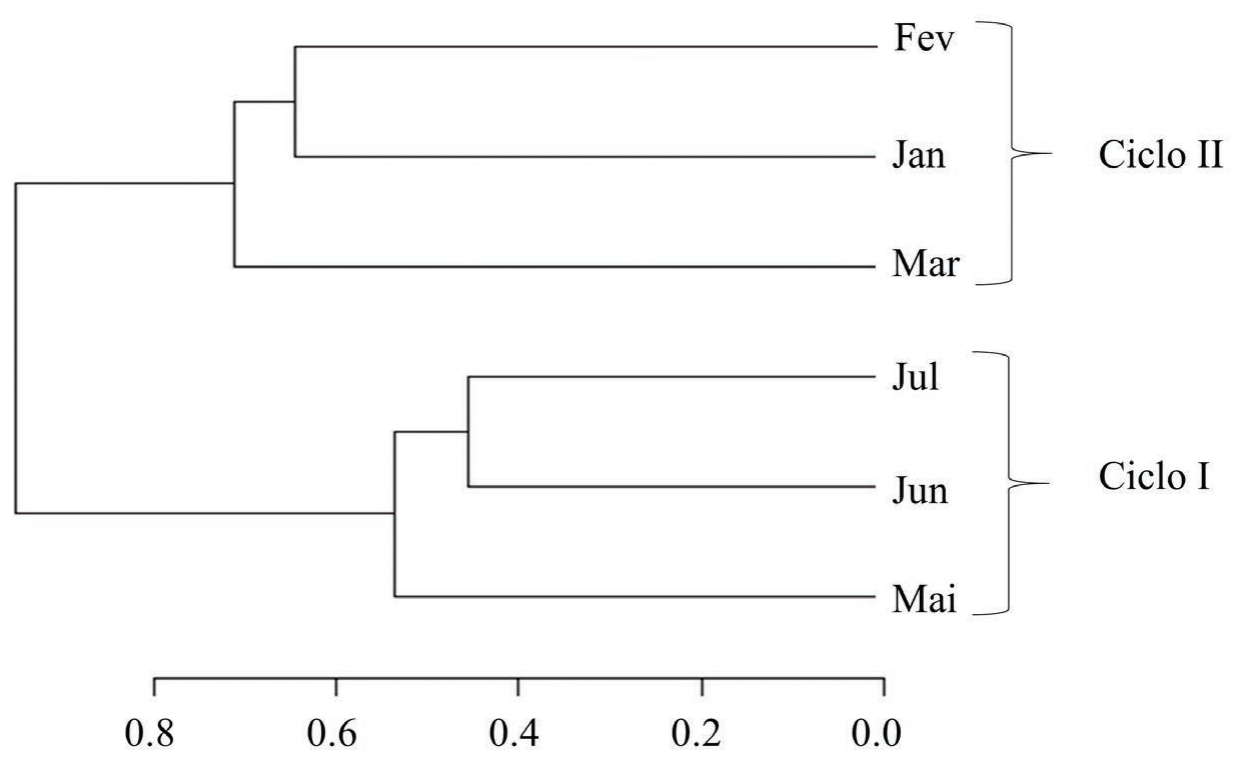

Figura 3: Dendrograma de similaridade entre os meses de amostragens na ilha das Marrecas no trecho baixo do rio Amazonas com base na abundância total de espécies coletadas (coeficiente de Bray-Curtis).

Figure 3: Dendrogram of similarity among the sampling months in the Ilha das Marrecas in the Lower Amazon River based on the total abundance of sampled species (Bray-Curtis coefficient).

estágios de pós-flexão (Tukey; $p=0,034$ ) e juvenil (Tukey; $p=0$,025) (Figura 5).

\section{DISCUSSÃO}

A elevada captura de espécies e indivíduos de diferentes estágios de desenvolvimento associados as macrófitas aquáticas reforça a premissa de que estes habitats desempenham importantes funções ecológicas em ecossistemas aquáticos lênticos de várzea. Estes ambientes são fundamentais por garantirem grande parte do desenvolvimento das larvas e juvenis de peixes ao longo de diferentes momentos do período hidrológico amazônico.

A predominância de Characiformes em bancos demacrófitastemsido registrada na Amazônia eem outras bacias hidrográficas da região Neotropical (Meschiatti et al. 2000, Sánchez-Botero e AraújoLima 2001, Sánchez-Botero et al. 2003, Agostinho et al. 2007, Pinheiro et al.2016, Araújo 2017). Em sua

Tabela 2: Diversidade de Shannon (H'), número de espécies (Riqueza), Dominância (D') e Equitabilidade de Pielou (J') da ictiofauna coletada nos bancos de macrófitas na Ilha das Marrecas durante os Ciclos I e II. Valores do Teste t independente. Valor de t (T), Graus de liberdade (gl), valor da significância $(p)$.

Table 2: Diversity of Shannon (H'), number of species (Richness), Dominance ( $\left.D^{\prime}\right)$ and Equitability of Pielou ( $\left.J^{\prime}\right)$ of ichthyofauna collected in macrophytes banks on the Island of Marrecas during Cycles I and II. Independent Test $t$ Values. Value of $t(T)$, Degrees of freedom (gl), value of significance ( $p$ ).

\begin{tabular}{lcccccccccc}
\hline \multirow{2}{*}{ Índices Ecológicos Ciclo II } & \multicolumn{3}{c}{ Ciclo I } & \multicolumn{3}{c}{ t } & \multirow{2}{*}{ gl } & \multirow{2}{*}{$p$} \\
\cline { 2 - 10 } & Maio & Junho & Julho & Janeiro & Fevereiro & Março & & & \\
\hline Shannon (H') & 2,91 & 2,7 & 2,41 & 1,76 & 1,59 & 1,91 & 5,3514 & 4 & $\mathbf{0 , 0 0 5 0}$ \\
Riqueza & 28 & 20 & 17 & 22 & 30 & 30 & $-1,3398$ & 4 & 0,2514 \\
Dominância & 0,07 & 0,08 & 0,11 & 0,24 & 0,35 & 0,23 & $-4,6346$ & 4 & $\mathbf{0 , 0 0 9 7}$ \\
Equitabilidade & 0,87 & 0,90 & 0,85 & 0,57 & 0,47 & 0,56 & 9,7253 & 4 & $\mathbf{0 , 0 0 0 6}$ \\
\hline
\end{tabular}




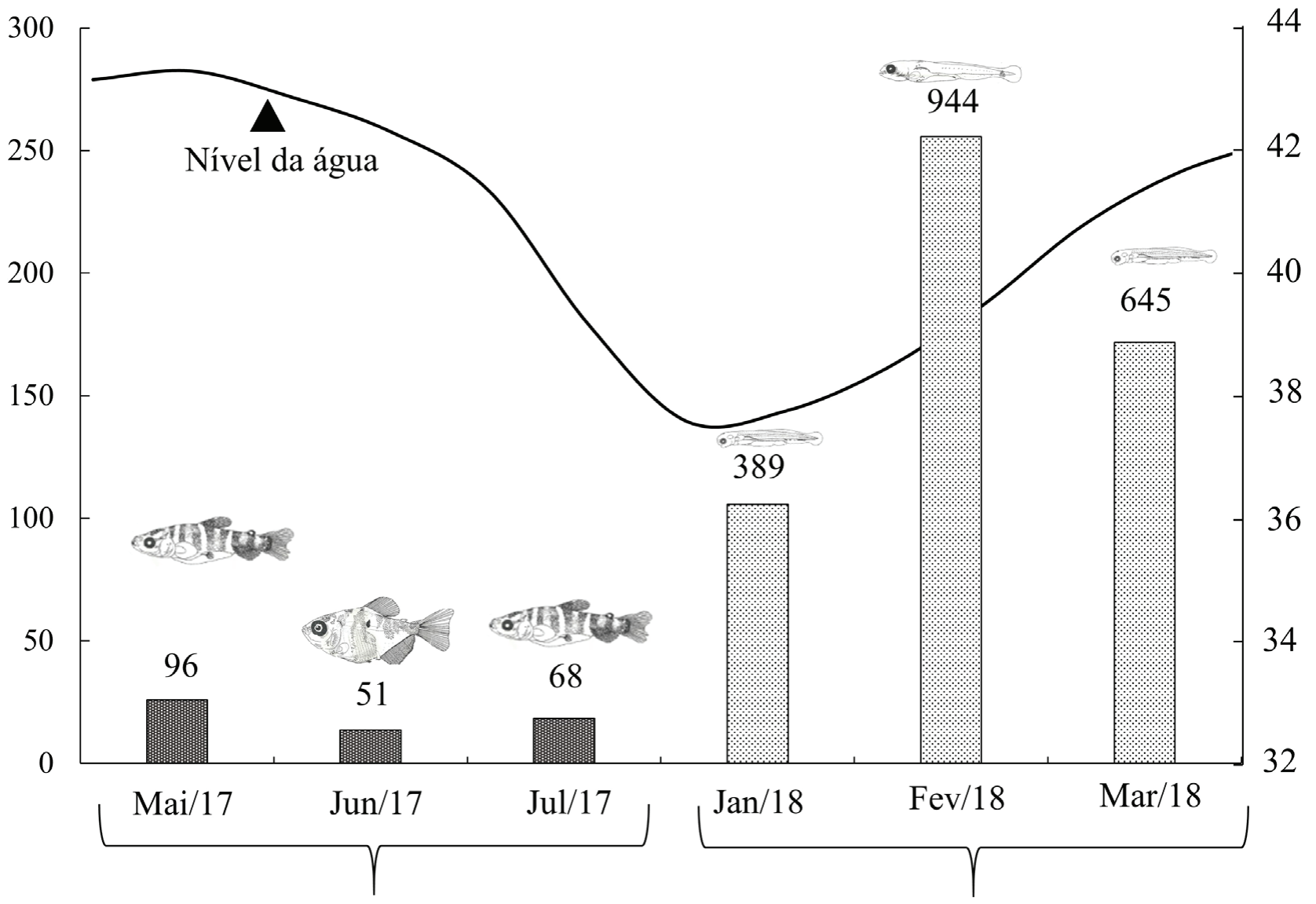

Figura 4: Variação temporal da densidade de indivíduos coletados durante os meses nos dois ciclos (I e II) de amostragens na Ilha das Marrecas, Santarém, Pará. Valores acima das colunas são referentes ao número total de indivíduos. (m.a.n.m = metros acima do nível do mar).

Figure 4: Temporal variation of the density of individuals sampled during the months of the two cycles (I and II) in the Marrecas Island, Santarém, state of Pará. Values above the columns refer to the total number of individuals. (m.a.n.m. = meters above sea level).

maioria, representada por indivíduos juvenis e/ou em fase inicial de desenvolvimento de espécies exploradas pelas frotas pesqueiras locais e por espécies de pequeno porte. Esta predominância pode estar relacionada, principalmente, com as características estruturais formadas pelas vegetações aquáticas, como a complexidade de habitat gerada por raízes, caules e folhas. Esses fatores físicos proporcionam condições ambientais favoráveis como disponibilidade de alimento e refúgio contra predadores e podem ser responsáveis por mais da metade da variação de espécies de peixes ao longo do habitat (Petry et al. 2003, Yu et al. 2016). Além do mais, larvas desta ordem têm sido predominantes em águas abertas de rios e lagos de várzea, durante o momento de enchente dos rios na Amazônia Ocidental (Zacardi 2014, Zacardi et al. 2017a, 2018, Ponte et al. 2019).
Este fato, provavelmente, justifica a grande captura de indivíduos de espécies migradoras e de interesse comercial em fase inicial de desenvolvimento nas amostragens durante $o$ ciclo II, período que engloba os primeiros meses do ano, ocasião de subida do nível das águas do sistema Solimões/Amazonas e de maior atividade reprodutiva das espécies de peixes dessa categoria na região (Zacardi et al. 2017a, b). A ilha das Marrecas é um importante local de retenção de ovos e larvas de peixes que derivam ao longo do canal principal do rio Amazonas (Zacardi et al. 2017b). Assim, as larvas conseguem encontrar nos bancos de macrófitas presentes na ilha condições propícias para o crescimento e desenvolvimento inicial.

Em geral, os habitats presentes nas áreas de várzea podem ser colonizados por larvas de peixes 


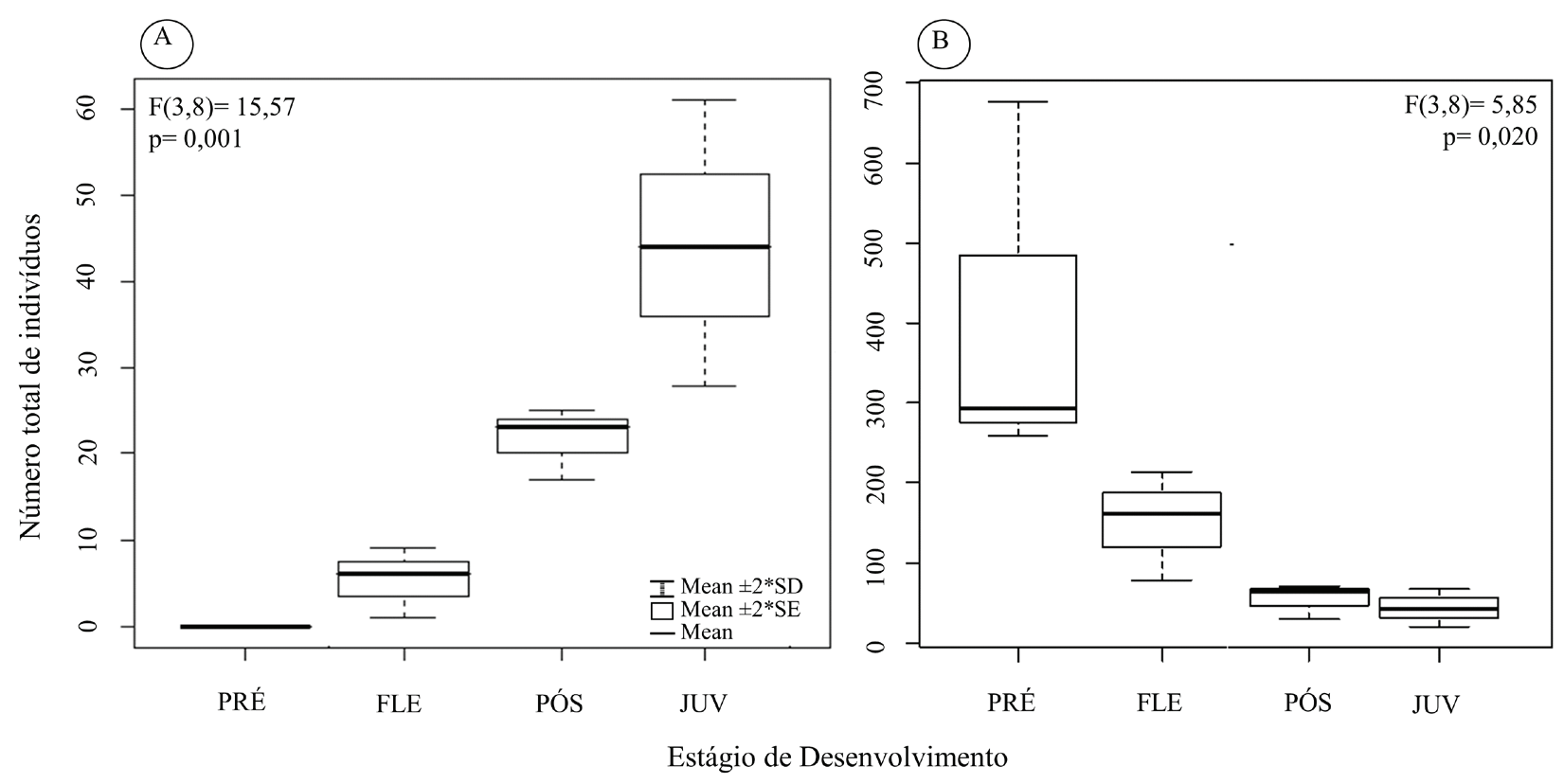

Figura 5: Variação dos estágios de desenvolvimento das larvas e juvenis associados aos bancos de macrófitas durante o ciclo I (A) e o ciclo II (B). Pré-flexão (PRÉ), flexão (FLE), pós-flexão (PÓS) e juvenil (JUV).

Figure 5: Variation of larval and juvenile development stages associated with macrophytes stands during cycle $I(A)$ and cycle II (B). Pre-flexion (PRÉ), flexion (FLE), post-flexion (POS) and juvenile (JUV).

que derivam por diversos canais, furos e lagos recém-formados da planície aluvial, incluindo as áreassubmersas dosinúmerosbancos demacrófitas aquáticas (Sanches-Botero et al. 2001, Zacardi 2014, Ponte et al. 2019). Estes habitas constituem locais propícios para alimentação, proteção contra predadores potenciais e recrutamento desses indivíduos. Além do mais, são considerados como áreas de berçário, fundamentais para o sucesso da manutenção das populações de peixes (Zacardi 2014, Goulding et al., 2018), principalmente para os pacus, do gênero Mylossoma, responsáveis pelas altas densidades de indivíduos registradas nos bancos de macrófitas durante o ciclo II.

Noperíodo deáguasaltas (cicloI-cheia/vazante), houve maior predominância de indivíduos juvenis ou adultos de espécies de pequeno porte como os caracídeos e poecilídeos. Estes resultados estão relacionados com o fato dos bancos de macrófitas aquáticas atuarem como habitat preferencial para esses grupos de pequenos peixes que necessitam se locomover apenas em distâncias curtas, a fim de encontrar outros estandes de macrófitas. Estas áreas são importantes locais de alimentação, uma vez que seus caules e raízes contribuem na produção de detritos (material particulado) e servem como substrato para a colonização da assembleia perifítica e abrigam ainda, uma grande quantidade de invertebrados que constituem importantes itens alimentares (Caffrey 1993, Junk \& Robertson 1997, Sanches-Botero et al. 2003).

Por outro lado, constituem-se também como zonas de refúgio, por proporcionarem uma eficiente barreira física e visual contra predadores a juvenis e peixes pequenos. Tais condições podem auxiliar no decréscimo da taxa de predação, possibilitando que organismos diversos, em termos morfológicos e funcionais, tornem-se aptos a explorar com sucesso estes habitats (Meschiatti et al. 2000, Pelicice et al. 2008, Pacheco \& Da-Silva 2009, Dibble \& Pelicice 2010).

Alguns trabalhos como os desenvolvidos por Jacobsen \& Perrow (1998) e Priyadarshana et al. (2001) já indicavam o papel das macrófitas como mediador nas interações predador-presa. No entanto, outros fatores podem ser responsáveis pela seleção do habitat pelos peixes além dos biológicos, tais como condições químicas e físicas, que proporcionam situações ideais para a colonização da ictiofauna em seus distintos momentos do ciclo de vida, tornando, portanto, os diversos ambientes de várzea como locais de berçário (Reynalte-Tataje 
et al. 2008, Zacardi et al. 2018) e desenvolvimento da comunidade íctica amazônica.

Das espécies capturadas, 30 possuem interesse econômico e são amplamente exploradas pela pesca comercial e de subsistência na região do Baixo Amazonas, como tambaqui (Colossoma macropomum), pacu comum (Mylossoma aureum), pacu manteiga (Mylossoma albiscopum), aracus (Schizodonfasciatus, Leporinusfasciatus, Rhytiodus microlepis), apapás (Pellona castelnaeana e $P$. flavipinnis), charutinhos (Anodus elongatus e Hemiodus spp.), sardinha comprida (Triportheus auritus), bagres como o bico-de-pato (Sorubim lima) e do gênero Pimelodus spp. (mandí) além de diversos ciclídeos como jacundás (Crenicichla spp.) e jurupari (Satanoperca sp.).

O elevado número de indivíduos de interesse econômico associado aos bancos de macrófitas na ilha das Marrecas, evidencia a importância desse componente de várzea na economia regional, uma vez que são capazes de auxiliar na renovação das espécies exploradas comercialmente no Baixo Amazonas. Tal aspecto, demonstra a eficiência dos bancos de macrófitas em auxiliar na manutenção dos estoques pesqueiros na região.

Essa eficiência na renovação dos estoques é essencial, principalmente, para os pacus ( $M$. albiscopum e $M$. aureum), espécies que possuem elevada importância na pesca comercial e de subsistência na região amazônica. Zacardi et al. (2018) ao investigarem abundância de larvas dessas espécies ao longo de dois anos, verificaram uma redução nas populações desses indivíduos, a qual esteve diretamente relacionada com as altas taxas de desembarque nos mercados e feiras regionais. Desse modo, as vegetações aquáticas podem contribuir significativamente na renovação desses estoques pesqueiros.

A baixa quantidade de indivíduos juvenis capturados, pode estar diretamente relacionada com o comportamento das espécies, como por exemplo a atividade trófica, fuga de predadores e a fase do ciclo de vida (Lucas \& Baras 2000). Além do mais, na fase juvenil o poder de natação dos peixes é maior em relação ao larval, podendo ter sido um forte influenciador na baixa abundância de espécimes juvenis. Nesta fase de desenvolvimento os indivíduos são mais propícios à fuga do apetrecho de coleta utilizado nas amostragens devido possuírem as estruturas de locomoção (ex. nadadeiras) mais desenvolvidas. Este fato pode ter sido um dos fatores que influenciaram na variação da abundância de indivíduos capturados.

De acordo com o teste de agrupamento realizado com base nas abundâncias totais de espécies, os meses de janeiro e fevereiro apresentaram $64 \%$ de similaridade, seguida pelo mês de março. Isso indica que ao longo de um ciclo hidrológico a composição de espécies pode ser a mesma e apresentar alterações ao longo dos demais momentos do ciclo. Essa variação na composição das espécies ao longo dos meses pode também ter sido influenciada não somente pelo ciclo hidrológico, mas também pelo nível da complexidade das vegetações aquáticas gerada pela morfologia radicular e estruturas foliares (Thomaz \& Cunha 2010). Juntas, essas duas condições maximizam as condições bióticas que favorecem a utilização das macrófitas pela ictiofauna como, por exemplo, a abundância de presas que variam de acordo com a sazonalidade aquática (Pelicice \& Agostinho 2006). Assim, foi verificado que o ciclo hidrológico é um dos responsáveis pela estruturação da composição ictiofaunística associada as macrófitas, uma vez que ao longo dos períodos analisados foi registrada a presença de indivíduos de diferentes estratégias ecológicas e estágios de desenvolvimento.

Os menores valores de diversidade e altos valores de dominância registrados ao longo do ciclo II podem ter sido influenciados diretamente pela elevada abundância das espécies do gênero Mylossoma e Triportheus. A qual pode estar relacionada a presença de recursos nutricionais associados às macrófitas, a atividade reprodutiva dessas espécies nas proximidades da área de estudo (Zacardi et al.2017a, Zacardi et al. 2018), bem como condições propícias para o desenvolvimento desses indivíduos na vegetação aquática, influenciando em uma alta dominância. Já os maiores valores de equitabilidade observados no ciclo I demonstram que as espécies apresentaram uma distribuição equitativa em relação a abundância de indivíduos. Essas diferenças de significância constatadas nos índices ecológicos são devidas a diversidade e riqueza de espécies em ambientes de várzea estarem associadas as fases do ciclo hidrológico (Amoros \& Bornette 2002, Hurd et al. 2016).

Portanto, por estar conectada ao longo de todo o ano com o canal principal do rio, a ilha das Marrecas funciona como uma área de retenção, 
no qual as larvas que estão à deriva ao longo do trecho inferior do rio Amazonas encontram nesse ambiente micro-habitats adequados, como os bancos de macrófitas, para o desenvolvimento ontogenético larval. Neste sentido, durante a enchente pode haver uma colonização de larvas de peixes nos bancos de macrófitas, e ao longo das demais fases do período hidrológico os indivíduos podem se desenvolver até adquirirem atributos morfológicos necessários para explorarem outros habitats. Deste modo, a presença de vegetação aquática parece ser de grande importância na estruturação, manutenção e desenvolvimento de larvas e juvenis de peixes durante os períodos de enchente e águas altas.

Por fim, os bancos de macrófitas apresentam capacidade para suportar espécies de diferentes categorias ecológicas, estratégias reprodutivas e de importância econômica para a região, indicando que este habitat é de grande relevância na manutenção e renovação dos estoques pesqueiros amazônicos. Logo, qualquer alteração, interferência ou distúrbios nas comunidades de macrófitas aquáticas, que não sejam naturais, podem afetar fortemente e acarretar transformações na estrutura e na dinâmica de colonização da comunidade de larvas e juvenis de peixes, com reflexos negativos no recrutamento de espécies.

\section{AGRADECIMENTOS}

Os autores agradecem aos demais colaboradores do Laboratório de Ecologia do Ictioplâncton e Pesca em Águas Interiores e a Coleção Ictiológica da Universidade Federal do Oeste do Pará pelo auxílio durante as coletas em campo e na identificação de alguns espécimes, respectivamente. À Universidade Federal do Oeste do Pará pela concessão da bolsa de Iniciação Científica ao primeiro autor para a execução do projeto e aos revisores anônimos que contribuíram com significativas sugestões para a melhoria deste artigo.

\section{REFERÊNCIAS}

Affonso, I. P., Gomes, L. C., Agostinho, A. A., Message, H. J., Latini, J. D. \& García-Berthou, E. 2016. Interacting effects of spatial gradients and fishing gears on characterization of fish assemblages in large reservoirs. Reviews in Fish Biology and Fisheries, 26(1), 71-81. DOI: 10.1007/s11160-015-9402-1.

Agostinho, A. A., Thomaz, S. M., Baltar, S. L. S. M. A. \& Gomes, L. C. 2002. Influence of aquatic macrophytes on fish assemblages structure of the Upper Paraná River floodplain (Brazil). In: Proceedings of the 11th EWRS International Symposium on Aquatic Weeds, Moliets et Maâ France. pp. 69-72.

Agostinho, A. A., Thomaz, S. M., Gomes, L. C. \& Baltazar, S. L. S. M. A. 2007. Influence of the macrophyte Eichornia azurea on fish assemblage of the upper Paraná River flooplain (Brazil). Aquatic Ecology, 41, 619-641. DOI: 10.1007/s10452-007-9122-2

Amoros, C \& Bornette, G. 2002. Connectivity and biocomplexity in waterbodies of riverine floodplains. Freshwater Biology, 47, 761-776. DOI: 10.1046/j.1365-2427.2002.00905.x

Araújo, A. F. 2017. Assembleia de peixes associada a bancos de macrófitas aquáticas no rio areias, reservatório da usina de Lajeado, Tocantins, Brasil: composição e distribuição. Dissertação de Mestrado. Universidade Federal do Tocantins. p. 45

Araújo-Lima, C. A. R. M. \& Donald, E. 1988. Número de vértebras de Characiformes do rio Amazonas e seu uso na identificação de larvas do grupo. Acta Amazonica, 18, 351-358. DOI: 10.1590/1809-43921988182358

Araújo-Lima, C. A. R. M., Kirovsky, A. L. \& Marca, A. G. 1993. As larvas dos pacus, Mylossoma spp. (Teleostei; Characidae), da Amazônia Central. Revista Brasileira de Biologia, 53, 297-306.

Arias, M. E., Wittmann, F., Parolin, P., MurrayHudson, M. \& Cochrane, T. A. 2018. Interactions between flooding and upland disturbance drives species diversity in large River floodplains. Hydrobiologia, 814, 5-17. DOI: 10.1007/s10750-016-2664-3

Barthem, R. B. \& Fabré, N. N. 2004. Biologia e diversidade dos recursos pesqueiros da Amazônia. In: M. L. Ruffino (Ed.), A pesca e os recursos pesqueiros na Amazônia brasileira. pp. 17-62. Manaus: Ibama/Provárzea.

Batista, V. S., Inhamuns, A. J., Freitas, C. E. C. \& Freire-Brasil, D. 1998. Characterization of the fishery in riverine communities in the LowSolimões/High-Amazon region. Fisheries 
Management and Ecology, 5, 419-435. DOI: 10.1046/j.1365-2400.1998.550419.x

Britisk, H. A., Silimon, K. Z. S. \& Lopes, B. S. 2007. Peixes do Pantanal: manual de identificação. 2a Ed. Brasília: Embrapa: p. 227.

Caffrey, J. M. 1993. Aquatic plant management in relation to Irish recreational fisheries development. Journal of Aquatic Plant Management, 31, 162-168.

Dibble, E. D. P. \& Pelicice F. M. 2010. Influence of aquatic plant-specific habitat on an assemblage of small neotropical floodplain fishes. Ecology of Freshwater Fish,19, 381-389. DOI: $10.1111 / \mathrm{j} .1600-0633.2010 .00420 . \mathrm{x}$

Ferreira, E. J. G., Zuanon, J. A. S. \& Santos, G. M. 1998. Peixes comerciais do médio Amazonas: região de Santarém, Pará. Brasília: IBAMA: p. 214.

Géry, J. 1977. Characoids of the world. New Jersey: TFH Publications: p. 672.

Gomes, L. C., Bulla, C. K., Agostinho, A. A., Vasconcelos, L. P. \& Miranda, L. E. 2012. Fish assemblage dynamics in a Neotropical floodplain relative to aquatic macrophytes and the homogenizing effect of a flood pulse. Hydrobiologia, 685, 97-107. DOI 10.1007/ s10750-011-0870-6

Goulding, M. 1980. The fishes and the forest: explorations in amazon natural history. Berkeley: University of Califórnia Press: p. 280 Goulding, M., Venticinque, E., Ribeiro, M. L. B., Barthem, R. B., Leite, R. G., Forsberg, B., Petry, P., Silva-Júnior, U. L., Ferraz, P. S. \& Cañas, C. 2018. Ecosystem-based management of Amazon fisheries and wetlands. Fish and Fisheries, 20, 138-158. DOI: 10.1111/faf.12328

Granado-Lorencio, C., Araújo-Lima, C. R. M. \& Lobón-Cerviá, J. 2005. Abundance distribution relationships in fish assembly of the Amazonas floodplain lakes. Ecography, 28, 515-520. DOI: 10.1111/j.09067590.2005.04176.x

Henderson, P. A. \& Hamilton, H. F. 1995. Standing crop and distribution of fish in drifting and attached floating meadow within an Upper Amazonian varzea lake. Journal of Fish Biology, 47, 266-276. DOI: 10.1111/j.10958649.1995.tb01894.x

Hurd, L., Sousa, R. G. C., Siqueira-Souza, F. K., Copper, G. J. \& Freitas, C. E. C. 2016.
Amazon floodplain fish communities: Habitat connectivity and conservation in a rapidly deteriorating environment. Biological Conservation, 195, 118-127. DOI: 10.1016/j. biocon.2016.01.005

Isaac, V. J., Almeida, M. C., Giarrizzo, T., Deus, C. P., Vale, R., Klein, G. \& Begossi, A. 2015. Food consumption as an indicator of the conservation of natural resources in riverine communities of the Brazilian Amazon. Anais daAcademia Brasileira de Ciências, 87(4), 22292242. DOI: $10.1590 / 0001-3765201520140250$

Jacobsen, L. \& Perrow, M. R. 1998. Predation risk from piscivorous fish influencing the diel use of macrophytes by planktivorous fish in experimental ponds. Ecology of Freshwater Fish, Denmark, 7(2), 78-86. DOI: 10.1111/ j.1600-0633.1998.tb00174.x

Junk, W. J. \& Robertson, B. 1997. Aquatic invertebrates. In: W. J. Junk (Ed.), The Central Amazonian floodplain: Ecology of a pulsing system. pp. 279-298. Heidelberg, Germany: Ecological Studies: p. 126.

Lucas, M. C. \& Baras, E. 2000. Methods for studying spatial behaviour of freshwater fishes in the natural environment. Fish and Fisheries, 1, 283-316. DOI: 10.1046/j.14672979.2000.00028.x

Magalhães, E. R. S., Yamamoto, K. C., Anjos, H. D., Loebens, S. C. \& Soares, M. G. M. 2015. Bancos de macrófitas aquáticas em lago de várzea: alimentação de duas espécies de peixes na região de Manaus, Amazonas, Brasil. Acta of Fisheries and Aquatic Resources, 3(1), 25-40. DOI 10.2312/ActaFish.2015.3.1.25-40.

Magurran, A. E. 2003. Measuring biological diversity. Oxford: Blackwell Press: p. 255.

Meschiatti, A. J., Arcifa, M. S. \& Fenerich-Verani, N. 2000. Fish communities associated with macrophytes in Brazilian floodplain lakes. Environmental Biology of Fishes, 58, 133-143.

Nakatani, K., Agostinho, A. A., Baumgartner, G., Bialetzki, A., Sanches, P. V., Makrakis, M. C. \& Pavanelli, C. S. 2001. Ovos e larvas de peixes de água doce: desenvolvimento e manual de identificação. Maringá: EDUEM: p. 378.

Oksanen, J., Blanchet, F. G., Friendly, M., Kindt, R., Legendre, P., McGlinn, D., Minchin, P. R., O'Hara, R. B., Simpson, G. L., Solymos, P., Stevens, M. H. H., Eduard-Szoecs E. \& Wagner, 
H. 2018. Vegan: Community Ecology Packpage $\mathrm{R}$ packpage version 2.5-2. https://CRAN.Rproject.org $/$ packpage. org $/$ package=vegan .

Orsi, M. L., Almeida, F. S., Swarc, A. C., Garcia, A. C., Garcia, D. A. Z., Vianna, N. C., \& Bialetzki, A. 2016. Ovos, larvas e juvenis de peixes da Bacia do rio Paranapanema: uma avaliação para a conservação. Londrina: Triunfal Gráfica e Editora: p. 136

Pacheco, E. B. \& Da-Silva, C. J. 2009. Fish associated with aquatic macrophytes in the Chacorore-Sinha Mariana Lake system and Mutum River, Pantanal of Mato Grosso, Brazil. Brazilian Journal of Biology, 69(1), 101-108. DOI: $10.1590 /$ S1519-69842009000100012

Pelicice, F. M. \& Agostinho, A. A. 2006. Feeding ecology of fishes associated with Egeria spp. patches in a tropical reservoir, Brazil. Ecology of Fresh Water Fish, 15, 10-19. DOI: 10.1111/j.1600-0633.2005.00121.x

Pelicice, F. M., Thomaz, S. M. \& Agostinho, A. A. 2008. Simple relationships to predict attributes of fish assemblages in patches of submerged macrophytes. Neotropical Ichthyology, 6, 543550. DOI: 10.1590/S1679-62252008000400001

Petry, P., Bayley, P. B. \& Markle, D. F. 2003. Relationships between fish assemblages, macrophytes and environmental gradients in the Amazon River Floodplain. Journal of Fish Biology, 63, 547-579. DOI: 10.1046/j.10958649.2003.00169.x

Pinheiro, D. T., Corrêa, J. M. S., Chaves, C. S., Campos, D. P. F., Ponte, S. C. S. \& Zacardi, D. M. 2016. Diversidade e distribuição da ictiofauna associada a bancos de macrófitas aquáticas de um lago de inundação amazônico, estado do Pará, Brasil. Acta of Fisheries and Aquatic Resources, 4(2), 59-70.

Ponte S. C. S., Oliveira L. S. \& Zacardi D. M. 2019. Temporal variation of fish larvae from a flooding lake as grant to environmental management. Journal of Applied HydroEnvironment and Climate, 1(1), 1-13.

Prado, K. L. L., Freitas, C. E. C. \& Oliveira, S. A. 2009. Assembleias de peixes associadas a diferentes bancos de macrófitas aquáticas em lagos de várzea do baixo rio Solimões. Revista Colombiana Ciencia Animal, 1(2), 185-201.

Prado, K. L. L., Freitas, C. E. C. \& Soares, M. G. M. 2010. Assembleias de peixes associadas às macrófitas aquáticas em lagos de várzea do baixo rio Solimões. Biotemas, 23(1), 131-142.

Priyadarshana, T., Asaeda, T. \& Manatunge, J. 2001. Foraging behaviour of planktivorous fish in artificial vegetation: the effects on swimming and feeding. Hidrobiologia, 442, 231-239.

R Core Team. 2018. R: A language and environment for statistical computing. R Foundation for Statistical Computing, Vienna, Austria. URL https://www.R-project.org/.

Reynalte-Tataje, D. A. \& E. Zaniboni-Filho. 2008. Biologia e identificação de ovos e larvas de peixes do alto rio Uruguai. In: E. ZaniboniFilho \& A. P. O. Nuñer (Eds.), Reservatório de Itá. Estudos ambientais, desenvolvimento de tecnologia e conservação da ictiofauna. pp. 229256 Florianópolis: Editora UFSC.

Saint-Paul, U., Zuarnon, J., Villacorta-Correa, M. A., Garcia, M., Fabre, N. N., Berger, U. \& Junk, W. J. 2000. Fish communities in central Amazonian white and blackwater floodplains. Environmental Biology of Fishes, 57, 235-250. DOI: 10.1023/A:1007699130333

Sánchez-Botero, J. I. \& Araújo-Lima, C. A. R. M. 2001. As macrófitas aquáticas como berçário para a ictiofauna da várzea do rio Amazonas. Acta Amazonica, 31, 437-447. DOI: 10.1590/1809-43922001313447.

Sánchez-Botero, J. I. S., Farias, M. L., Piedade, M. T. \& Garcez, D. S. 2003. Ictiofauna associada às macrófitas aquáticas Eichhornia azurea (SW.) Kunth. e Eichhornia crassipes (Mart.) Solms. no lago Camaleão, Amazônia Central, Brasil. Acta Scientiarum. Biological Sciences, 25(2), 369375. DOI: 10.4025 /actascibiolsci.v25i2.2026

Sánchez-Botero, J. I., Araújo-Lima, C. A. R. M. \& Garcez, D. S. 2008. Effects of types of aquatic macrophyte stands and variations of dissolved oxygen and of temperature on the distribution of fishes in lakes of the Amazonian floodplain. Acta Limnológica Brasileira, 20(1), 45-54.

Santos, G. M., Mérona, B., Juras, A. A. \& Jégu, M. 2004. Peixes do baixo rio Tocantins: 20 anos depois da usina hidrelétrica Tucuruí. Brasília: Eletronorte: p. 216.

Santos, G. M., Ferreira, E. J. G. \& Zuanon, J. A. S. 2006. Peixes Comerciais de Manaus. Manaus: Ibama/Provárzea: p. 144.

Schiesari, L., Zuanon, J., Azevedo-Ramos, C., Garcia, M., Gordo, M., Messias, M. \& Monteiro, E. 2003. 
Macrophyte rafts as dispersal vectors for fishes and amphibians in the Lower Solimões River, Central Amazon. Journal of Tropical Ecology, 19, 333-336. DOI: 10.1017/S0266467403003365

Soares, M. G. M., Almeida, R. G. \& Junk, W. J. 1986. The trophic status of the fish fauna in Lago Camaleão, a macrophyte dominated floodplain lake in the middle Amazon. Amazoniana, 9(4), 511-526.

Soares, M. G. M., Costa, E. L., Siqueira-Souza, F. K., Anjos, H. D. B., Yamamoto, K. C. \& Carvalho, C. E. F. 2008. Peixes de lagos do Médio Rio Solimões. Manaus: Instituto Piatam: p. 160.

Soares, M. G. M., Costa, E. L., Siqueira-Souza, F. K., Anjos, H. D. B. \& Yamamoto, K. C. 2011. Peixes de Lagos do Médio Rio Solimões. 2a Ed. Manaus: Reggo Edições: p. 176.

Soares, M. G. M., Freitas, C. E. C. \& Oliveira, A. C. B. 2014. Assembleias de peixes associadas aos bancos de macrófitas aquáticas em lagos manejados da Amazônia Central, Amazonas, Brasil. Acta Amazonica, 44(1), 143-152. DOI: 10.1590/S0044-59672014000100014

Thomaz, S. M \& Cunha, E. R. 2010. The role of macrophytes in habitat structuring in aquatic ecosystems: methods of measurement, causes and consequences on animal assemblages' composition and biodiversity. Acta Limnologica Brasiliensia, 22(2), 218-236. DOI: 10.4322/ actalb.02202011

Winer, B. J., Brown, D. R. \& Michels, K. M. 1991. Statistica principals in experimental design. $3^{\mathrm{a}}$ Ed. New York: Mcgraw-hill: p. 907.

Yamamoto, K. C., Soares, M. G. M. \& Freitas, C. E. C. 2004. Alimentação de Triportheus angulatus (Spix \& Agassiz, 1829) no lago Camaleão, Manaus, AM, Brasil. Acta Amazonica, 34(4), 653659. DOI: 10.1590/S0044-59672004000400017

Yu, J., Liu, Z., Ele, H., Zhen, W., Guan, B., Chen, F., Li, K., Zhong, P., Teixeira-de-Melo, F. \& Jeppesen, E. 2016. Submerged macrophytes facilitate dominance of omnivorous fish in a subtropical shallow lake: implications for lake restoration. Hydrobiologia, 775 (1), 97-107

Zacardi, D. M. 2014. Abundância e distribuição espaço-temporal de ovos e larvas de peixes de interesse econômico no médio rio Solimões e baixo rio Japurá, Amazônia Central, Brasil. Tese de Doutorado. Universidade Federal do Pará. p. 120.
Zacardi, D. M., Bittencourt, S. C. S., Nakayama, L. \& Queiroz,H.L.2017a.Distributionofeconomically important fish larvae (Characiformes, Prochilodontidae) in the Central Amazonia, Brazil. Fisheries Management and Ecology, 24, 01-09. DOI: 10.1111/fme.12222

Zacardi, D. M., Ponte, S. C. S., Ferreira, L. C., Lima, M. A. S., Silva, A. J. S. \& Chaves, C. S. 2017b. Diversity and spatio-temporal distribution of the ichthyoplankton in the lower Amazon River, Brazil. Biota Amazônia, 7 (2), 12-20. DOI: 10.18561/2179-5746

Zacardi, D. M., Ponte, S. C. S., Chaves, C. S., Oliveira, L. S. \& Cajado, R. A. 2018. Variação interanual no recrutamento de larvas de Mylossoma (Characidae; Characiformes) no Baixo Amazonas, Brasil. Acta of Fisheries and Aquatic Resources, 6(1), 17-28.

Zar, J. H. (1999). Biostatistical analysis. $4^{\text {a }}$ Ed. New Jersey: Prentice-Hall: p. 663.

Submitted: 6 Abril 2019

Accepted: 5 February 2020

Published on line: 12 February 2020 Associate Editor: José Luís Novaes 